\title{
EVALUATING DYNAMIC ERROR OF A TREADMILL AND THE EFFECT ON MEASURED KINETIC GAIT PARAMETERS: IMPLICATIONS AND POSSIBLE SOLUTIONS
}

\author{
Alessandro Garofolini ${ }^{1}$, Simon Taylor $^{1}$ and Julien Lepine ${ }^{1,2}$ \\ ${ }^{1}$ Victoria University, Institute of Sport Exercise and Active Leaving (ISEAL), Ballarat \\ Road, Footscray, Melbourne, Victoria 3011, Australia \\ ${ }^{2}$ University of Cambridge, Department of Engineering, Trumpington St, Cambridge, CB2 \\ 1PZ, UK
}

Corresponding author email: j.lepine@eng.cam.ac.uk

Corresponding author phone: +44 1223332868

\begin{abstract}
The dynamic properties of instrumented treadmills influence the force measurement of the embedded force platform. We investigated these properties using a frequency response function, which evaluates the ratio between the measured and applied forces in the frequency domain. For comparison, the procedure was also performed on the gold-standard ground-embedded force platform. A predictive model of the systematic error of both types of force platform was then developed and tested against different input signals that represent three types of running patterns. Results show that the treadmill structure distorts the measured force signal. We then modified this structure with a simple stiffening frame in an attempt to reduce measurement error. Consequently, the overall absolute error was reduced (-22\%), and the error in force-derived metrics was also sufficiently reduced: $-68 \%$ for average loading rate error and $-80 \%$ for impact peak error. Our procedure shows how to measure, predict, and reduce systematic dynamic error associated with treadmill-installed force platforms. We suggest this procedure should be implemented to appraise data quality, and frequency response function values should be included in research reports.
\end{abstract}

KEYWORDS: biomechanics; gait analysis; calibration; ground reaction force; running.

WORD COUNT: 3973 


\section{INTRODUCTION}

2 Force platforms are an essential measurement device in many biomechanical studies, from which kinetic

3 parameters are derived to evaluate gait. As an adjunct to the common ground-installed force platform sensor $\left(\mathrm{G}_{F S}\right)$,

4 the treadmill-installed force platform sensor $\left(\mathrm{T}_{F S}\right)$ is becoming popular in gait research laboratories (Dierick, Penta,

5 Renaut, \& Detrembleur, 2004; Riley et al., 2008; Riley, Paolini, Della Croce, Paylo, \& Kerrigan, 2007). Given that

6 kinetic parameters depend on accurate force signal measurements (Pàmies-Vilà, Font-Llagunes, Cuadrado, \&

7 Alonso, 2012; Silva \& Ambrósio, 2004), data quality and research integrity relies upon the known degree of

8 measurement error associated with these force-instrumented treadmills. The precision of a force measurement

9 device is dependent upon the inherent natural frequency of its structure. Depending on the mass and stiffness of a

10 treadmill structure, and on the force sensor size (Dierick et al., 2004), treadmill dynamic behavior may generate

11 mechanical vibrations and mode shapes at specific frequencies (natural frequencies) that could approach the

12 frequency content of applied forces from human gait and create artefacts in the measurements. While the ground-

13 installed force platforms have natural frequencies much higher than the frequency content of the exerted force

14 (Antonsson \& Mann, 1985), the natural frequencies of the treadmill installed platforms have been reported to be as

15 low as $16 \mathrm{~Hz}$ in some cases (Draper, 2000) that is within the frequency content of normal gait (reported as 35-

$1650 \mathrm{~Hz}$ (Antonsson \& Mann, 1985; Blackmore, Willy, \& Creaby, 2016)), affecting the accuracy of the measured

17 force by the strain gauges (force sensors) (Willems \& Gosseye, 2013). Nowadays, there is a rise in research that

18 uses parameters derived by treadmill-installed force platforms data for training and retraining (rehabilitative)

19 interventions, in both sport (Crowell \& Davis, 2011) and clinical settings (Van den Noort, Steenbrink, Roeles, \&

20 Harlaar, 2015), as well as for development of new technologies (Mooney \& Herr, 2016). Although accurate

21 measurement of force data is paramount, it is not common practice to include an independent report on the

22 frequency response and the expected measurement error of the forces.

23 The error inherent within force measurement is best detected and evaluated from frequency domain analysis

24 (Gruber, Boyer, Derrick, \& Hamill, 2014; Gruber, Davis, \& Hamill, 2011). Therefore, this study will evaluate the

25 Ground Reaction Force signal (GRF) in the frequency domain and describe its harmonic contents, as per (White, 
Agouris, \& Fletcher, 2005). The inherent error in the GRF created by the natural frequency of the treadmill is not a random noise that may disappear by taking the average or integration of measured signals across gait cycles.

28 Instead, this error is systematic; it has the same effect on each measurement episode. Bias created by the natural 29 frequency is not related to the magnitude of signal noise that can be overcome by smoothing process that produces 30 a best-fit line (De Bièvre, 2009), but it is related to the degree of difference between the measured and smoothed 31 signal and the true signal (Menditto, Patriarca, \& Magnusson, 2007). Therefore, bias is an essential feature to 32 consider when comparing measurements obtained across different force platform systems.

33 At the authors best knowledge, only one study included the issue of natural frequency testing on instrumented treadmills (Sloot, Houdijk, \& Harlaar, 2015). They presented a new approach to test the performance of treadmills, assessing the accuracy of forces and center of pressure, including assessment of the natural frequency. However, they did not explore the effect of low natural frequencies on force signals, nor propose any solution to improve treadmill performance. Our study continues upon this theme by outlining a standardized method to evaluate natural

38 frequencies and their effect on measurement bias. The three aims of this study were: i) to evaluate measurement

39 bias (systematic error) of an instrumented treadmill using a test for frequency-dependent behavior of a force 40 platform; ii) to develop and evaluate a model that is designed to predict measurement bias of the force platform 41 frequency response; and iii) to reduce measurement bias of an instrumented treadmill.

\section{METHODS}

43 The aims were addressed in three stages. Stage 1 assessed the dynamic behavior of the instrumented treadmill using 44 Frequency Response Function (FRF) (Rao \& Yap, 2011). This was achieved by evaluating the signal frequency 45 ratio between two interacting force measurement devices. We used a hammer installed force sensor $\left(\mathrm{H}_{F S}\right)$ to apply an impact force to a treadmill-installed force platform sensor $\left(\mathrm{T}_{F S}\right)$, and to a ground-installed force platform sensor

$47\left(\mathrm{G}_{F S}\right)$. Stage 2 evaluated a model that was developed to predict the dynamic behavior of the treadmill (refer to (Rao \& Yap, 2011) for more details on the mathematical procedure used to develop the model). Stage 3 assessed a solution to improve the dynamic behavior of $\mathrm{T}_{F S}$ by altering the support structure of the treadmill. We then assessed the dynamic behaviour of the new $\mathrm{TW}_{F S}$ using the predictive model. 


\section{$51 \quad$ Stage 1}

\section{Analysis of treadmill frequency response}

53 The Fourier transform represents any signal - such as the force signal - as a sum of periodic waveforms (e.g. sine 54 functions). Each waveform is characterized by a frequency $(\omega)$, an amplitude $(A)$ and a phase $(\phi)$. This allows 55 investigation of how the signal's amplitude and phase vary for any given frequency. The systematic error of the 56 force platforms $\left(\mathrm{T}_{F S}\right.$ or $\mathrm{G}_{F S}$ ) can be represented in the frequency domain using a FRF. The FRF is a frequency 57 dependent modulation system that alters the frequency properties of the input signal (Figure 1). For example, the 58 amplitude $\left(A_{i}\right)$ and phase $\left(\phi_{i}\right)$ of the input signal pass through the modulation function, where the signal is 59 transformed into an output signal with new amplitude $\left(A_{o}\right)$ and phase $\left(\phi_{o}\right)$.

60

$$
A_{F R F}(j \omega) \angle \phi_{F R F}(j \omega)=\frac{A_{o}(j \omega) \angle \phi_{o}(j \omega)}{A_{i}(j \omega) \angle \phi_{i}(j \omega)}
$$


72 Now, it is possible to look at how the system (FRF) reacts for each frequency of the input signal using the 73 following transfer function estimator:

$$
F R F(\omega)=\frac{F P(\omega)}{H(\omega)}
$$

where $F P(\omega)$ is the Fourier transform of the force platform signal and $H(\omega)$ is the Fourier transform of the hammer signal. The change in amplitude and phase caused by the modulation system can then be represented as:

$$
\begin{aligned}
A_{F R F}(\omega) & =|F R F(\omega)| \\
\phi_{F R F}(\omega) & =\angle F R F(\omega)
\end{aligned}
$$

80 where $A_{F R F}$ defines how the system affects the amplitude of the input signal (in absolute terms) for any given

81 frequency, and $\phi_{F R F}$ defines how the system affects the phase of the input signal for any given frequency.

\section{Measurement}

83 The $\mathrm{H}_{F S}$ was composed of a high precision force sensor (PCB Piezotronics, 218A) fixed on the head of a modified

84 hammer, so-called impact hammer. The $\mathrm{G}_{F S}$ were embedded into a ground-installed force platform (BP600900TT, 85 AMTI, USA). The $\mathrm{T}_{F S}$ were embedded into a treadmill-installed force platform (DBCEEWI, AMTI, USA). The 86 impact hammer has been calibrated using a known mass and accelerometer (Waltham \& Kotlicki, 2009) and 87 connected to a 2 channel charge amplifier (Rion, UV-16). The devices were synchronized using Nexus data 88 acquisition system (Oxford Metrics Ltd, Oxford, UK) at a sample frequency of $2000 \mathrm{~Hz}$. The $\mathrm{H}_{F S}$ has a flat 89 response up to $1000 \mathrm{~Hz}$ (Appendix A), therefore it provides an accurate measure of the force applied to the 90 platforms. The ratio between the output from platform force sensors and the $\mathrm{H}_{F S}$ shows how the measurement is 91 affected by the dynamic behavior of the system. When the response is $1 \mathrm{~N} / \mathrm{N}$, it means that the force measured by 92 both instruments perfectly match. 
Using the hammer we generated a set of 20 vertical impacts at five locations on each platform (four corners and the platform center). The average magnitude of the impacts was $100.2 \pm 39.7 \mathrm{~N}$, which is the linear range of the force platform $(0-8800 \mathrm{~N})$ meaning that the measured FRF is valid for any force below $8800 \mathrm{~N}$. The FRF linearity was validated with a coherence function which was above 0.90 between $5-200 \mathrm{~Hz}$ (Randall, 2008). Data were exported to Matlab (Math Works Inc., USA) for FRF analysis, averaging the 20 impacts to achieve adequate coherence function between 0 and $100 \mathrm{~Hz}$. In order to evaluate the dynamic behavior of the treadmill, the FRF was computed from the force signals of force platforms and hammer using the so-called H1 estimator (Rocklin, Crowley, \& Vold, 1985), which reduces the effect of the measurement noise in the force platforms signal, therefore:

$$
\operatorname{FRF}(\omega)=\frac{P_{F P H}}{P_{H H}}
$$

Where $P_{F P H}$ is the cross-spectrum between the force platform and the hammer signals, and $P_{H H}$ is the autospectrum of the $\mathrm{H}_{F S}$ signal (Randall, 2008). Amplitude and phase were then evaluated to investigate the occurrence of the first mode of vibration (i.e. natural frequency).

\section{Stage 2}

\section{Predictive Model}

The FRF of the measurement devices (e.g. force platform on the treadmill) represents, in the frequency domain, how a force measurement is distorted at every frequency by the dynamic behavior of the measurement device (e.g. natural frequency of the structure). An ideal measurement device would have a flat FRF throughout its frequency range which means that there would be no amplification nor delay between the real input (e.g. applied force) and reading (e.g. measured force).

Effect of the amplification and delay on the measurement can be assessed in the time domain using a predictive model. To do so, the first step was to transform the FRF into the time domain using the inverse Fast Fourier transform (Randall, 2008). The transformed FRF is known as the Impulse Response Function (IRF). The reading 
115 on the measurement device, $y_{F P}(t)$, in response to a certain input, $x(t)$, can be predicted by convolving the IFR with $116 \quad x:$

$$
y_{F P}(t)=\operatorname{IRF}(t) * x(t) \triangleq \int \operatorname{IRF}(\tau) x(t-\tau) d \tau
$$

117 where $\tau$ is a time lag integration variable.

118 The accuracy of the treadmill and ground-installed force-platforms measurements can be assessed be comparing the 119 predicted response of both measurement devices for different inputs. We selected three archetypal signals that 120 represent the vertical component of typical ground reaction force vectors (VGRF) generated by humans when 121 running (data collected in a previous experiment). These archetypes had distinct impact transients associated with 122 low, medium, and high loading (Figure 2).

126 Stage 3

127 Application and evaluation of a stiffening frame

128 The treadmill-installed force platforms are supported by a framework structure of steel beams (Figure 3). The 129 rectangular shape of the treadmill frame lays upon four feet posted at the corners. To stiffen the long axis of the frame 130 and increase the natural frequency, we positioned two wooden support bearers under each long side of the treadmill 131 frame (Figure 3, appendix B). To evaluate the bias of the new system, TWFS response was modelled and tested using 132 the three archetypal signals as input. Bias is reported as root mean squared error (RMSE). The natural frequency 133 didn't shift between tests and the coherence function was close to one, which suggests that the supports behave 134 linearly throughout all the tests. 


\section{$138 \quad$ RESULTS}

139 Treadmill frequency response

140 Figure 4 presents the amplitude (a) and phase shift (b) features of the FRFs produced from the hammer test on the

141 three measurement systems: $\mathrm{G}_{F S}, \mathrm{~T}_{F S}$, and $\mathrm{TW}_{F S}$.

145 For the amplitude, a FRF $<1$ implies there is an underestimation of the signal at that frequency, whereas a FRF $>1$

146 implies that there is an overestimation at that frequency. For instance, at $30 \mathrm{~Hz}$ the ratio between the applied force

147 and the measured one is 1.6, which means the measured force at $30 \mathrm{~Hz}$ is $37 \%$ greater than what it is in reality (i.e.

148 the force applied by the hammer). At $32 \mathrm{~Hz}$ there is a $10 \%$ increase with respect to $30 \mathrm{~Hz}$. Thus, between $32 \mathrm{~ms}$

149 and 33 ms of the loading phase, the measured signal will show a $10 \%$ increase in the first peak force that does not

150 exist in reality. At $40 \mathrm{~Hz}$ (ratio 0.68 ) the measurement by the $\mathrm{T}_{F S}$ will underestimate the force by $47 \%$.

151 The $\mathrm{T}_{F S}$ FRF presents two peaks at $32 \mathrm{~Hz}$ and $55 \mathrm{~Hz}$; whereas the $\mathrm{G}_{F S}$ shows the relatively flat response that is 152 expected from a gold-standard force measurement device (Figure 4a). After applying wooden bearers to the

153 treadmill, the first natural frequency shifted from 32 to $36 \mathrm{~Hz}$. For the phase, $\mathrm{T}_{F S}$ shows two main shifts at the two 154 natural frequencies (32 and $55 \mathrm{~Hz}$ ) and $\mathrm{TW}_{F S}$ has also a phase shift in correspondence of its first natural frequency 155 (36 Hz). In contrast, the $\mathrm{G}_{F S}$ shows no phase shift among the analyzed frequencies. 
158 Table 1 lists the level of agreement between the three archetypal signals and the model-predicted VGRF signals 159 derived from the FRF. The degree of overlap between the measured and archetypal signals for the three different 160 types of impact intensity and force sensor type is shown in Figure 5. The measurement error of the $\mathrm{G}_{F S}$ increases as 161 loading intensity increases while, the lowest error for the $\mathrm{T}_{F S}$ was at Medium load (52.5 $\mathrm{N}$ ) and the highest value 162 was at High loading (127.8 N), representing a $243 \%$ relative increase. $\mathrm{TW}_{F S}$ follows a similar trend to $\mathrm{T}_{F S}$. The 163 largest difference between $\mathrm{T}_{F S}$ and $\mathrm{TW}_{F S}$ was in High loading condition with a reduction in RMSE of 48\%. Overall 164 the $\mathrm{TW}_{F S}$ displays less error (-22\%) compared to the $\mathrm{T}_{F S}$. The modified frame reduced the error in the variables 165 related to the impact transient, such as average loading rate (ALR) and impact peak. The TWFS exhibits an error 3166 times lower in the ALR (a reduction of 68 percentage points), and an error 5-times lower in the impact peak (a 167 reduction of 80 percentage points; see Table 1).

171 Figure 5 (a-c) shows the three archetypal signals ( $\mathrm{a}$ - low; b - medium; c - high) compared against the predicted 172 force reading for the $G_{F S}, T_{F S}$ and $T_{F S}$. Figure 5 (d-f) represents the raw error for each condition. Main error for 173 the $\mathrm{T}_{F S}$ is in the first half of stance at high loading with an evident oscillatory behavior that decays over time. $\mathrm{TW}_{F S}$ 174 consistently overestimates the force measurement in early stance and underestimates it from mid stance forward. $175 \mathrm{G}_{F S}$ almost perfectly measures force applied in any loading condition. 
The general aim of this study was to evaluate the force measurement bias from a typical $\mathrm{T}_{F S}$ by comparing it

181 against a 'gold standard' $\mathrm{G}_{F S}$. The force reading from the $\mathrm{G}_{F S}$ is precise across a range of analyzed frequencies (1$100 \mathrm{~Hz}$ ), whilst the signal from the $\mathrm{T}_{F S}$ has some measurement bias. Any applied force to the $\mathrm{T}_{F S}$ that is above $10 \mathrm{~Hz}$ will either over- or under-estimate the true magnitude of the applied force and this measurement error will depend on the frequency content of the applied force.

The measurement error of the treadmill followed a different trend compared to the ground-installed force platform. While the $\mathrm{G}_{F S}$ showed a consistent increase with the loading intensity, the $\mathrm{T}_{F S}$ was inconsistent between these three archetypal signals. This is explained by the number and position of the treadmill's natural frequencies. The $\mathrm{G}_{F S}$ has a very high first natural frequency (> $500 \mathrm{~Hz}$ ), while the treadmill has two natural frequencies at approximately 32 bearers does not eliminate the natural frequencies, the trend is similar for the $\mathrm{TW}_{F S}$.

The first natural frequency of the treadmill was identified at $59 \mathrm{~Hz}$ prior to shipping (Appendix C). This suggests that the measured first natural frequency $(32 \mathrm{~Hz})$ was either not identified by the manufacturer, or the testing conditions were different. For instance, the soft elastic floor covering the ground (Mondo®) in our laboratory creates a compliant substrate of the treadmill-floor interface, which may have changed modes in the frequency bandwidth of interest. To further investigate the reasons for these discrepancies, a full modal analysis of the treadmill including several degree of freedom must be performed in different laboratory environments (e.g. floor structure, and mounting conditions). This type of systematic study would highlight how the dynamic behaviors of the system depend on its boundary conditions and establish general guideline for instrumented-treadmill installation.

202 The position where the measurements are made could also affect the number of natural frequencies appearing in the 203 frequency response function. If the excitation or the measurement has been made on a 'node' of a mode shape, the natural frequency of this mode doesn't appear on the FRF. As the tests presented in this paper were conducted at 
the point where the runner most commonly hits the platforms (i.e. its center), we ensured that all the relevant natural frequencies were measured. After modelling the FRF for the $\mathrm{G}_{F S}, \mathrm{~T}_{F S}$ and the adapted $\mathrm{TW}_{F S}$, we then compared their output force measurement with archetypal signals. While the $\mathrm{G}_{F S}$ seems to be more consistent in measurement error between loading intensities, the $\mathrm{T}_{F S}$ behaves differently depending on the type of VGRF profiles (Figure 5): it may be the case that the frequency content of the input signal is actually increasing as the loading profile of the VGRF increases. VGRF with high loading profile has a frequency content close to a

211 resonance frequency of the treadmill, therefore the measured force signal is amplified. Instead, when the VGRF

212 curve becomes smoother the frequency content changes - reduce - moving away from a resonance frequency; as a

213 result, the signal is minimally amplified due to the structural damping.

214 Due to the low natural frequencies of the treadmill, the $\mathrm{T}_{F S}$ VGRF profile degenerates, leading to errors in 215 measures of gait particulars associated with the impact transient (Table 1). For instance, the recorded signals by the $216 \mathrm{~T}_{F S}$ show that there can be errors in impact transient parameters of up to $12 \%$. Accurate measurement of impact 217 transient parameters is important for clinical evaluation of running performance and risk of injuries (Davis, Milner, 218 \& Hamill, 2004; Milner, Ferber, Pollard, Hamill, \& Davis, 2006). Moreover, results from running retraining studies 219 (Crowell, Milner, Hamill, \& Davis, 2010) aiming to reduce the impact transient may be affected by the dynamic 220 behavior of the instrumented treadmill. The measurement bias could be either systematic or random - because it is 221 dependent upon frequency; hence if a person applies different load intensities the observed error could vary 222 (under/over) between foot contacts within a trial. Therefore, pre-post intervention differences may be partially contributed by the bias associated with the dynamic (vibratory) behavior of the treadmill. For many future studies using instrumented treadmills, researchers could evaluate the confidence they have in their data by using the FRF and IRF method. Indeed this is performed by manufacturers prior to shipping, however, this evaluation also needs to be conducted in the lab setting.

It is worth noticing that measurement errors - related to the dynamic behavior of the treadmill - will pass undetected when error evaluation techniques are employed with conventional static calibrations (Gill \& O'Connor, 1997; Hsieh, Lu, Chen, Chang, \& Hung, 2011). The results from the dynamic validation method performed in this study demonstrates the effect that a $\mathrm{T}_{F S}$ can have on the data quality within a biomechanics lab, and raises the 
231 necessity to include such an evaluation procedure as regular practice prior to the reporting of data. The evaluation 232 of the modified $\mathrm{TW}_{F S}$ is indicative of why a $\mathrm{T}_{F S}$ should be tested in its specific environment and condition. The 233 application of supports underneath the body of the treadmill showed an overall improvement of the ratio between 234 input (hammer) and output (force platform), reducing the measurement error of the VGRF. Although the natural 235 frequency has been increased slightly (from $32 \mathrm{~Hz}$ to $36 \mathrm{~Hz}$ ), the reduction of the error is remarkable. For instance, 236 at $30 \mathrm{~Hz}$ the ratio decreased from 1.60 to 1.15, reducing the $37 \%$ artificial increase in force recording to just $13 \%$.

237 When comparing the amount of measurement bias (RMSE) and the change in loading variables across the different 238 loading conditions, the modified $\mathrm{TW}_{F S}$ shows a smaller average error (Table 1). Although a benchmark of an acceptable error limit will vary according to derived parameters, we can consider a level of error equivalent to that 240 of the ground embedded force platform as the gold standard benchmark. Achieving this will require improvement 241 in two areas: (i) mathematical models of the frequency response, and (ii) engineering a stiffening frame comparable 242 to a ground embedded force platform. A mathematical model will minimize the effect of systematic error; while an 243 improved frame structure will increase resonance frequency and provide a more reliable measurement of high 244 frequency forces.

245 Indeed, the effect of systematic artifact will have a greater impact on certain users and their analyses, while others 246 might find these levels acceptable. For example, the ground reaction force orientation may be sufficiently altered to 247 affect joint kinetic parameters, particularly the hip joint moments (where a combination of both kinematic and 248 kinetic errors would exist). In another context, the appeal of using instrumented treadmills is that they accommodate analyses that require long continuous data sets. However, analyses that quantify time-series behavior of gait parameters (e.g. (Dingwell, John, \& Cusumano, 2010; Hausdorff et al., 1996) should be cautious when

251 considering similar analyses on gait parameters measured from instrumented treadmills, particularly impact 252 transient.

253 An alternative method to avoid sensor natural frequency related error is to use a digital low-pass filter. Commonly, 254 in running studies, force signals are low-pass filtered with a cut-off frequency of $50 \mathrm{~Hz}$ (Baggaley, Willy, \& 255 Meardon, 2017; Cheung \& Rainbow, 2014; Kulmala, Avela, Pasanen, \& Parkkari, 2013) with some using 100 Hz 256 (Hobara, Sato, Sakaguchi, Sato, \& Nakazawa, 2012). As the frequency content of the force signal recorded during 
running can reach frequencies up to $50 \mathrm{~Hz}$ (Blackmore et al., 2016; Shorten \& Mientjes, 2011), any cut-off

258 frequency lower than $50 \mathrm{~Hz}$ will necessarily delete part of the true signal. In our case, as the first natural frequency started affecting the signal at $10 \mathrm{~Hz}$, a lower cut-off frequency (i.e. $6 \mathrm{~Hz}$ ) would be needed to remove the amplification effect caused by the treadmill dynamic behavior, however, it will also smooth every sharp change in 261 the signal (i.e. rising portion of the GRFv). Therefore, when applying a low-pass filter to the force signal, the user should appreciate the effect of three influential factors: (1) the natural frequency of the treadmill; (2) the typical

263 frequency content of the force signal being recorded (i.e. influence of different types of impact); and (3) the type of 264 bias that the treadmill's dynamic behavior has on the force signal. In this study we showed how to address those issues with a rather simple test. Results will give confidence not only on the validity of the force signal, but also on the adequacy of low-pass filter cut-off frequency.

267 The main limitation of this study is the generalizability of our results. As the laboratory environment affects the natural frequency, the error found and solution proposed is only applicable to our treadmill. However, with this study we highlight the need of ensuring appropriate system quality check and report of measurement associated error which should be a priority for any biomechanical laboratory. Although our method was able to raise the natural frequency of the treadmill, it improved force reading accuracy without suppressing the bias. However, the procedure presented highlights that an evaluation of $\mathrm{T}_{F S}$ measurements performed in the frequency domain provide

273 sensitive characteristics of the force signal that can expose any presence of systematic error - this form of measurement error would otherwise be undetected through time domain procedures. Such an evaluation should always be performed in situ, that is, in the specific environment and condition in which the treadmill is used, and results should accompany any reported data for quality assurance.

\section{Conflict of interest statement}

The authors have no personal financial conflict of interests related to this study. 


\section{REFERENCES}

Antonsson, E. K., \& Mann, R. W. (1985). The frequency content of gait. Journal of biomechanics, 18(1), 39-47.

Baggaley, M., Willy, R., \& Meardon, S. (2017). Primary and secondary effects of real-time feedback to reduce vertical loading rate during running. Scandinavian journal of medicine \& science in sports, 27(5), 501-507.

Blackmore, T., Willy, R. W., \& Creaby, M. W. (2016). The high frequency component of the vertical ground reaction force is a valid surrogate measure of the impact peak. Journal of biomechanics, 49(3), 479-483. doi:https://doi.org/10.1016/j.jbiomech.2015.12.019

Cheung, R. T., \& Rainbow, M. J. (2014). Landing pattern and vertical loading rates during first attempt of barefoot running in habitual shod runners. Human movement science, 34, 120-127.

Crowell, H. P., \& Davis, I. S. (2011). Gait retraining to reduce lower extremity loading in runners. Clinical Biomechanics, 26(1), 78-83.

Crowell, H. P., Milner, C. E., Hamill, J., \& Davis, I. S. (2010). Reducing impact loading during running with the use of real-time visual feedback. Journal of Orthopaedic \& Sports Physical Therapy, 40(4), 206-213.

Davis, I., Milner, C. E., \& Hamill, J. (2004). Does increased loading during running lead to tibial stress fractures? A prospective study. Medicine and Science in Sports and Exercise, 36(5).

De Bièvre, P. (2009). The 2007 International Vocabulary of Metrology (VIM), JCGM 200: 2008 [ISO/IEC Guide 99]: Meeting the need for intercontinentally understood concepts and their associated intercontinentally agreed terms. Clinical biochemistry, 42(4), 246-248.

Dierick, F., Penta, M., Renaut, D., \& Detrembleur, C. (2004). A force measuring treadmill in clinical gait analysis. Gait \& Posture, 20(3), 299-303.

Dingwell, J. B., John, J., \& Cusumano, J. P. (2010). Do humans optimally exploit redundancy to control step variability in walking? PLoS Comput Biol, 6(7), e1000856.

Draper, E. R. (2000). A treadmill-based system for measuring symmetry of gait. Medical Engineering \& Physics, 22(3), 215-222.

Gill, H., \& O'Connor, J. (1997). A new testing rig for force platform calibration and accuracy tests. Gait \& Posture, 5(3), 228-232.

Gruber, A. H., Boyer, K. A., Derrick, T. R., \& Hamill, J. (2014). Impact shock frequency components and attenuation in rearfoot and forefoot running. Journal of Sport and Health Science, 3(2), 113-121.

Gruber, A. H., Davis, I. S., \& Hamill, J. (2011). Frequency content of the vertical ground reaction force component during rearfoot and forefoot running patterns. Medicine \& Science in Sports \& Exercise, 43(5), 60.

Hausdorff, J. M., Purdon, P. L., Peng, C., Ladin, Z., Wei, J. Y., \& Goldberger, A. L. (1996). Fractal dynamics of human gait: stability of long-range correlations in stride interval fluctuations. Journal of Applied Physiology, 80(5), 1448-1457.

Hobara, H., Sato, T., Sakaguchi, M., Sato, T., \& Nakazawa, K. (2012). Step frequency and lower extremity loading during running. Int J Sports Med, 33(4), 310-313. doi:10.1055/s-0031-1291232 
Hsieh, H.-J., Lu, T.-W., Chen, S.-C., Chang, C.-M., \& Hung, C. (2011). A new device for in situ static and dynamic calibration of force platforms. Gait \& Posture, 33(4), 701-705.

Kulmala, J.-P., Avela, J., Pasanen, K., \& Parkkari, J. (2013). Forefoot strikers exhibit lower running-induced knee loading than rearfoot strikers. Med Sci Sports Exerc, 45(12), 2306-2313.

Menditto, A., Patriarca, M., \& Magnusson, B. (2007). Understanding the meaning of accuracy, trueness and precision. Accreditation and Quality Assurance: Journal for Quality, Comparability and Reliability in Chemical Measurement, 12(1), 45-47.

Milner, C. E., Ferber, R., Pollard, C. D., Hamill, J., \& Davis, I. S. (2006). Biomechanical factors associated with tibial stress fracture in female runners. Medicine and Science in Sports and Exercise, 38(2), 323.

Mooney, L. M., \& Herr, H. M. (2016). Biomechanical walking mechanisms underlying the metabolic reduction caused by an autonomous exoskeleton. Journal of neuroengineering and rehabilitation, 13(1), 4.

Pàmies-Vilà, R., Font-Llagunes, J. M., Cuadrado, J., \& Alonso, F. J. (2012). Analysis of different uncertainties in the inverse dynamic analysis of human gait. Mechanism and machine theory, 58, 153-164.

Randall, R. B. (2008). Spectral Analysis and Correlation. In D. Havelock, S. Kuwano, \& M. Vorländer (Eds.), Handbook of Signal Processing in Acoustics (pp. 33-52). New York, NY: Springer New York.

Rao, S. S., \& Yap, F. F. (2011). Mechanical vibrations (Vol. 4): Prentice Hall Upper Saddle River.

Riley, P. O., Dicharry, J., Franz, J., Croce, U. D., Wilder, R. P., \& Kerrigan, D. C. (2008). A kinematics and kinetic comparison of overground and treadmill running. Medicine and Science in Sports and Exercise, 40(6), 1093.

Riley, P. O., Paolini, G., Della Croce, U., Paylo, K. W., \& Kerrigan, D. C. (2007). A kinematic and kinetic comparison of overground and treadmill walking in healthy subjects. Gait \& Posture, 26(1), 17-24.

Rocklin, G. T., Crowley, J., \& Vold, H. (1985). A comparison of H1, H2, and Hv frequency response functions. Paper presented at the Proceedings of the 3rd international Modal Analysis Conference.

Shorten, M., \& Mientjes, M. I. V. (2011). The 'heel impact' force peak during running is neither 'heel' nor 'impact' and does not quantify shoe cushioning effects. Footwear Science, 3(1), 41-58. doi:10.1080/19424280.2010.542186

Silva, M. P., \& Ambrósio, J. A. (2004). Sensitivity of the results produced by the inverse dynamic analysis of a human stride to perturbed input data. Gait \& Posture, 19(1), 35-49.

Sloot, L., Houdijk, H., \& Harlaar, J. (2015). A comprehensive protocol to test instrumented treadmills. Medical Engineering \& Physics, 37(6), 610-616.

Van den Noort, J. C., Steenbrink, F., Roeles, S., \& Harlaar, J. (2015). Real-time visual feedback for gait retraining: toward application in knee osteoarthritis. Medical \& Biological Engineering \& Computing, 53(3), 275-286.

Waltham, C., \& Kotlicki, A. (2009). Construction and calibration of an impact hammer. American Journal of Physics, 77(10), 945-949.

White, R., Agouris, I., \& Fletcher, E. (2005). Harmonic analysis of force platform data in normal and cerebral palsy gait. Clinical Biomechanics, 20(5), 508-516.

Willems, P. A., \& Gosseye, T. P. (2013). Does an instrumented treadmill correctly measure the ground reaction forces? Biology open, 2(12), 1421-1424. 


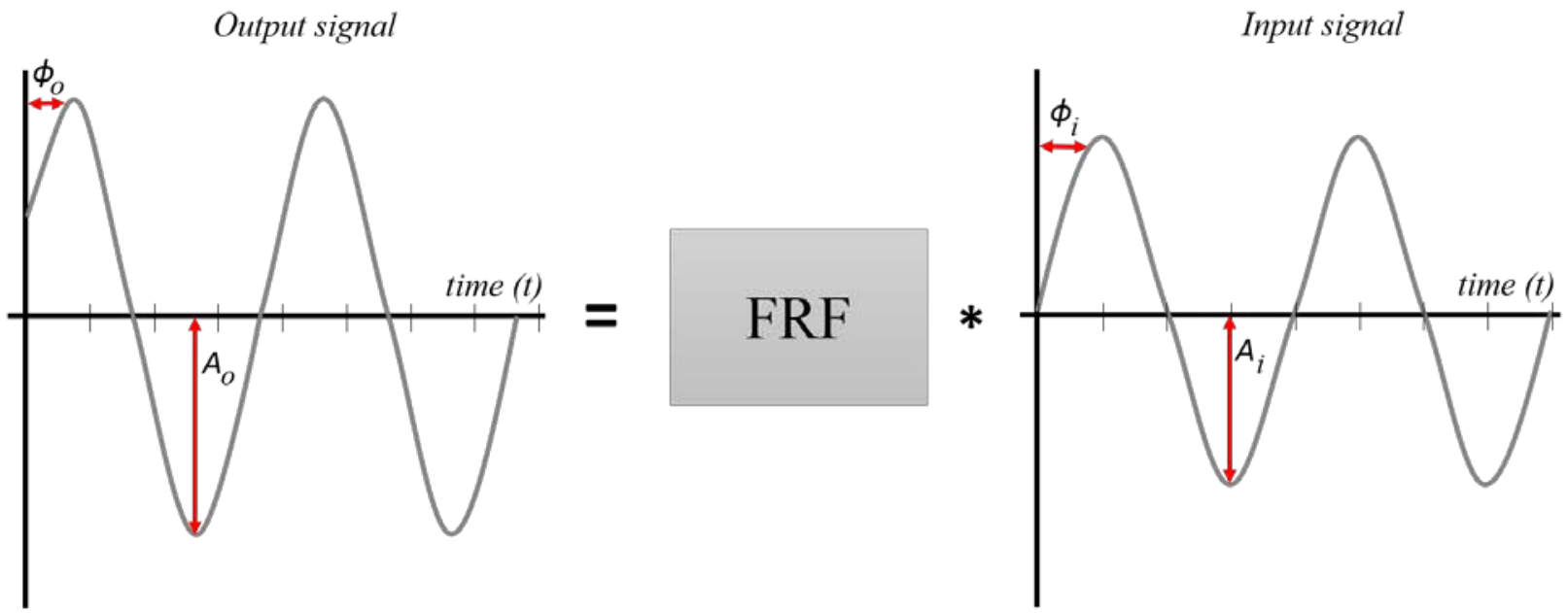

Figure 1 Response of a linear time-invariant system to a sinusoidal input (right). The steady state output (left) depends on the characteristics of the system (FRF). 

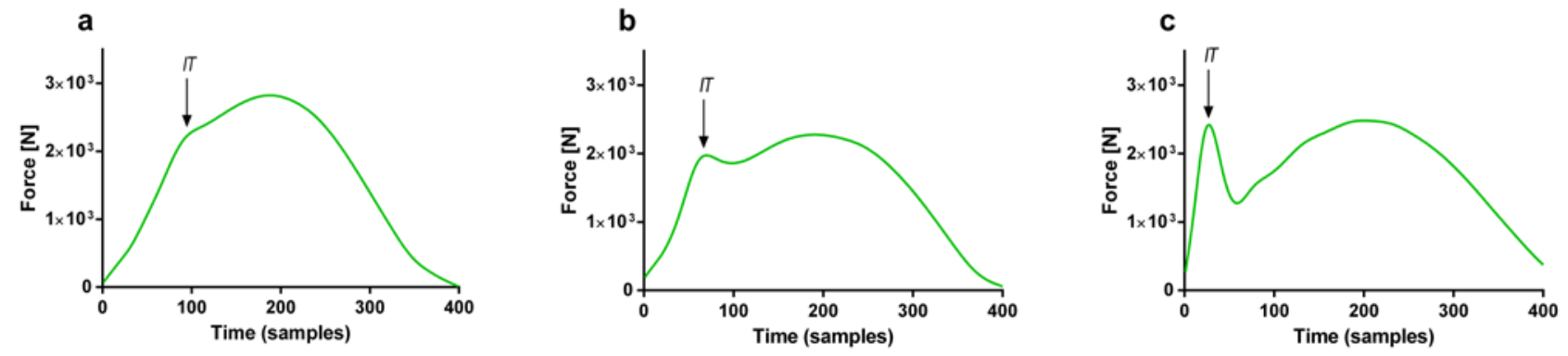

Figure 1: GRF archetypal signals with different impact transient properties. The intensity of the loading is low (a), moderate (b) and high (c); IT indicates the Impact Transient. 

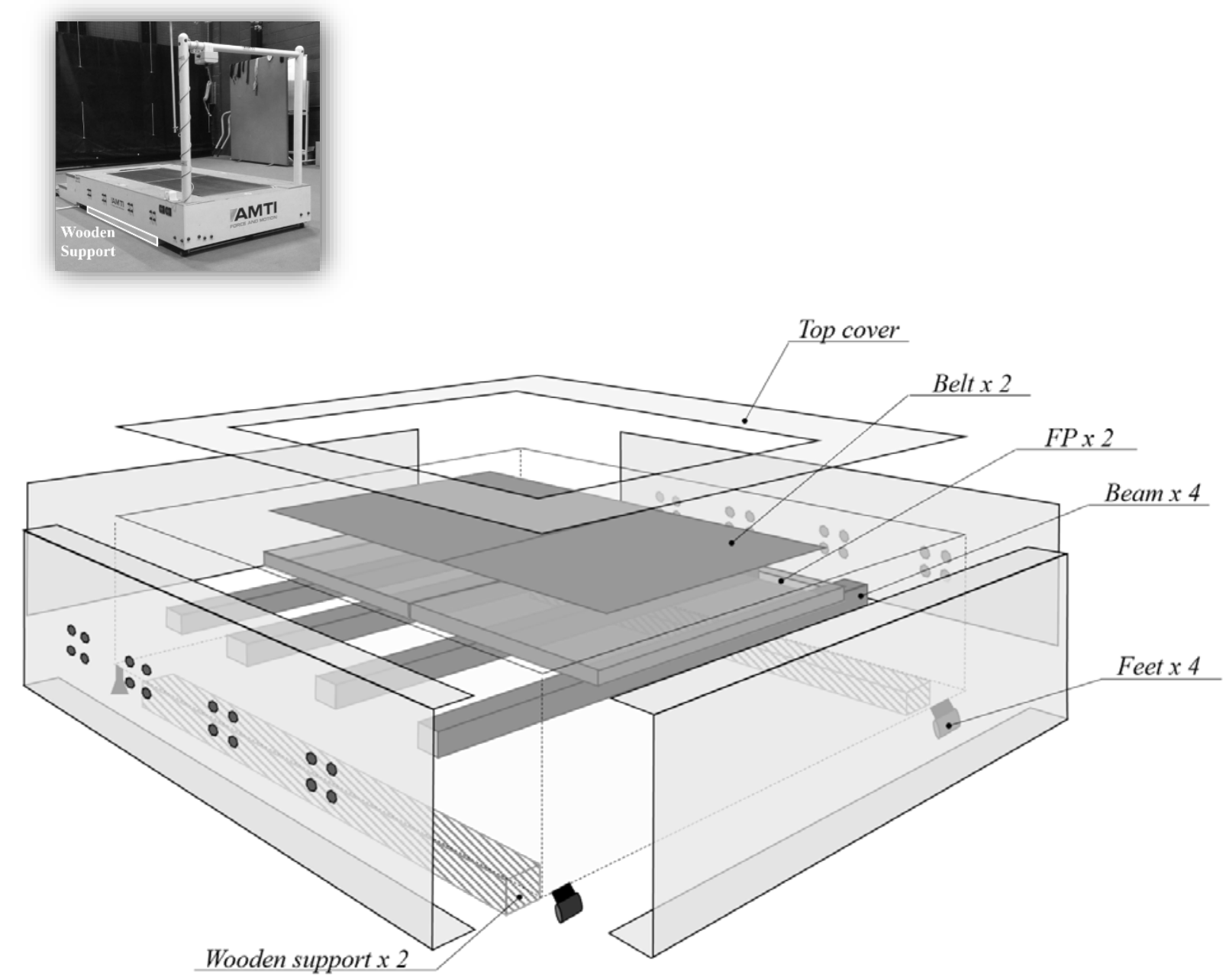

Figure 3 Structural components of the instrumented treadmill. Wooden supports were added underneath the lateral sides of the treadmill frame to improve overall stiffness of the device. Treadmill was resting on the wooden supports instead of on the four legs during the experiment." 

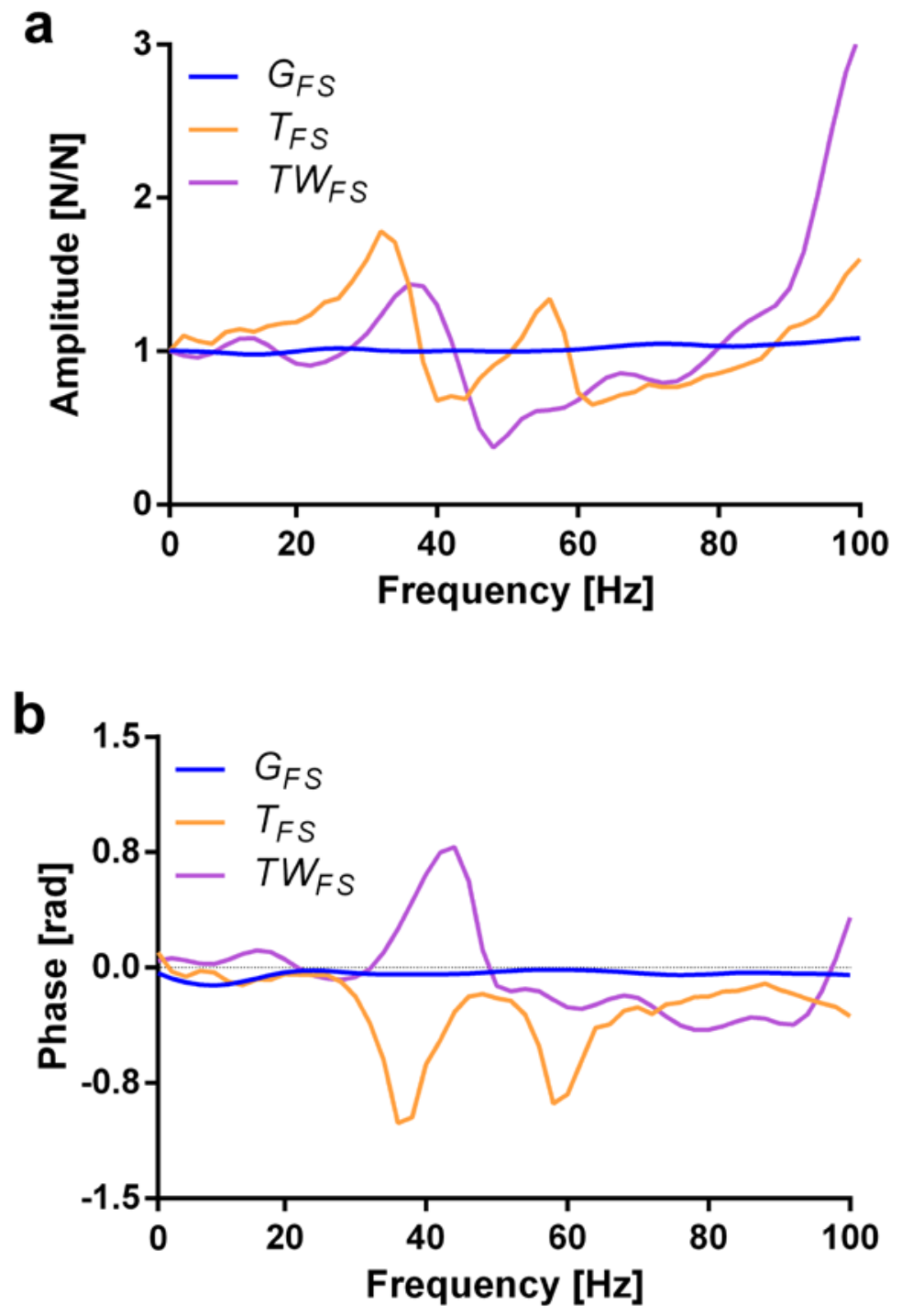

Figure 4. Frequency Response Function test displayed in the Amplitude (a) and phase (b) domain. FRF outcomes of the three hammer tests are over-ground sensor ( $G \mathrm{Fs}$, blue), treadmill sensor ( $T_{\mathrm{Fs}}$, orange), and treadmill with wood sensor (TW $\mathrm{FS}$, purple). 

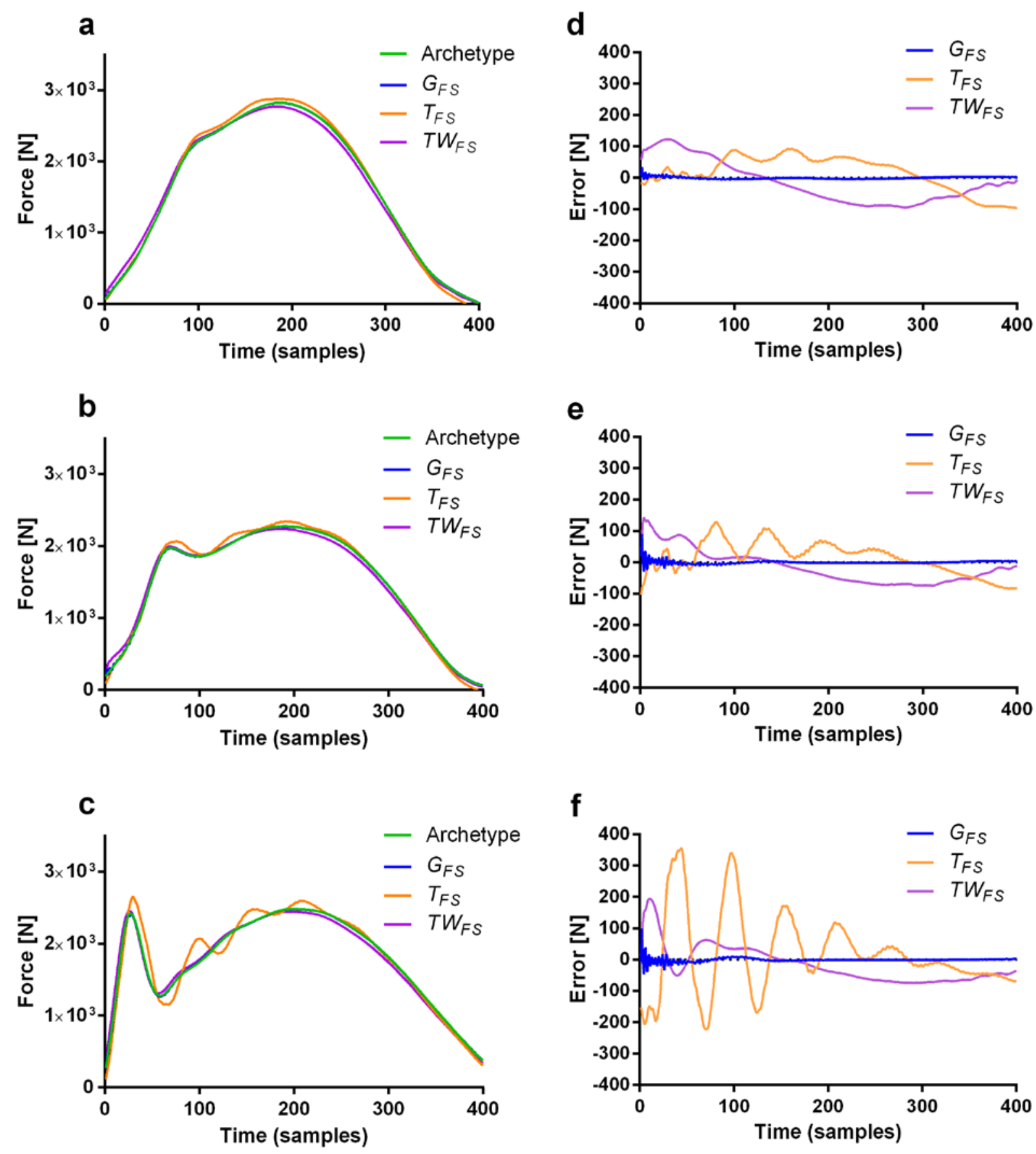

Figure 5. Archetypal VGRF signals from over-ground running with low loading (a), medium loading (b), and high loading (c). Archetypal VGRF signal (green) is compared against over-ground model-prediction (GFs blue), treadmill modelprediction ( $T_{\mathrm{FS}}$ orange), and new treadmill configuration (with wood bearers) model-prediction (TWFs purple). Error for each model is reported for low loading (d), medium loading (e), and high loading $(f)$. 
Table 1 Root mean squared error (RMSE) is reported as a measure of bias. The error of over-ground force platform sensor $\left(\mathrm{G}_{F S}\right)$, treadmill-installed force platform sensor $\left(\mathrm{T}_{F S}\right)$, and adapted treadmill ( $\left.\mathrm{TW}_{F S}\right)$ are reported for low loading (Low), medium loading (Med) and high loading profiles (High). The average (AVG) is also reported. RMSE is reported as raw values [N], percentage of peak force, and percentage of mean force. Average loading rate (ALR) and Impact peak are reported as percentage change from the archetypal VGRF signals. ALR was computed between 20-90\% of impact peak.

\begin{tabular}{|c|c|c|c|c|}
\hline & \multicolumn{3}{|c|}{ Loading pattern } & \multirow[b]{2}{*}{ AVG } \\
\hline & Low & Med & High & \\
\hline \multicolumn{5}{|l|}{ RMSE [N] } \\
\hline$G_{\mathrm{FS}}$ & 3.9 & 7.0 & 8.4 & 6.4 \\
\hline$T_{\mathrm{FS}}$ & 56.7 & 52.5 & 127.8 & 79.0 \\
\hline$T W_{\mathrm{FS}}$ & 68.4 & 54.9 & 60.7 & 61.3 \\
\hline \multirow{2}{*}{\multicolumn{5}{|c|}{$\begin{array}{l}\text { RMSE \% Peak } \\
\text { Force }\end{array}$}} \\
\hline $\begin{array}{l}\text { Force } \\
\quad G_{\mathrm{FS}}\end{array}$ & & & & \\
\hline$T_{\mathrm{FS}}$ & 2.0 & 2.3 & 5.2 & 3.2 \\
\hline$T W_{\mathrm{FS}}$ & 2.4 & 2.4 & 2.4 & 2.4 \\
\hline \multicolumn{5}{|l|}{$\begin{array}{l}\text { RMSE \% Mean } \\
\text { Force }\end{array}$} \\
\hline$G_{\mathrm{FS}}$ & 0.2 & 0.5 & 0.5 & 0.4 \\
\hline$T_{\mathrm{FS}}$ & 3.5 & 3.5 & 7.2 & 4.7 \\
\hline$T W_{\mathrm{FS}}$ & 4.2 & 3.6 & 3.4 & 3.7 \\
\hline \multicolumn{5}{|l|}{$\operatorname{ALR}(\Delta \%)$} \\
\hline$G_{\mathrm{FS}}$ & -2.0 & -3.8 & -1.3 & 2.4 \\
\hline$T_{\mathrm{FS}}$ & 1.8 & 12.3 & 3.7 & 5.9 \\
\hline$T W_{\mathrm{FS}}$ & -1.5 & 3.4 & 0.8 & 1.9 \\
\hline \multicolumn{5}{|l|}{$\begin{array}{l}\text { IMPACT PEAK } \\
(\Delta \%)\end{array}$} \\
\hline$G_{\mathrm{FS}}$ & -0.4 & 0.0 & 0.4 & 0.3 \\
\hline$T_{\mathrm{FS}}$ & 4.1 & 4.8 & 9.2 & 6 \\
\hline$T W_{\mathrm{FS}}$ & 1.1 & 1.3 & 1.1 & 1.2 \\
\hline
\end{tabular}


Revisions

-C- Rev \# 5104

e) $m$.

\section{DYNAMIC PERFORMANCE}

Range:

Maximum Force:

Compression

Tension

Compression

Tension

Resolution

Sensitivity (nominal)

Resonant Frequency

Rise Time

Amplitude Non-Linearity

Stiffness

ENVIRONMENTAL

Temperature Range

Temperature Coefficient

Vibration

Shock

ELECTRICAL

Capacitance

Insulation Resistance at Room Temperature

Polarity:

\section{MECHANICAL}

Dimensions:

Weight

Housing

Connector

Connector Orientation

Mating Connector Required

Sealing

Mounting Thread

Mounting Torque

\author{
Ib $[\mathrm{kN}]$ \\ lb $[\mathrm{kN}]$ \\ $\mathrm{lb}[\mathrm{kN}]$ \\ lb [kN] \\ Ib [kN] \\ $\mathrm{pC} / \mathrm{lb}[\mathrm{pC} / \mathrm{kN}]$ \\ $\mathrm{kHz}$ \\ $\mu \mathrm{sec}$ \\ $\%$ F.S. \\ $\mathrm{lb} / \mu$ in $[\mathrm{kN} / \mu \mathrm{m}]$ \\ ${ }^{\circ} \mathrm{F}\left[{ }^{\circ} \mathrm{C}\right]$ \\ $\% / F[\% / \mathrm{C}]$ \\ $\pm \mathrm{gk}\left[ \pm \mathrm{m} / \mathrm{s}^{2} \mathrm{pk}\right]$ \\ $\pm \mathrm{g} \mathrm{pk}\left[ \pm \mathrm{m} / \mathrm{s}^{2} \mathrm{pk}\right]$ \\ $\mathrm{pF}$
ohms
compression
tension \\ in \\ [mm] \\ oz [grams] \\ material \\ type \\ position \\ type \\ type \\ size \\ in-lb $[\mathrm{N}-\mathrm{mm}]$
}

[4]

$10[1,757]$

-400 to +400 [-240 to +204$]$

$0.01[0,018]$

2000 [19 620]

10000 [98 100]

12

$1 \times 10^{12}$

Negative

Positive

$0.625 \times 0.625$

$[15,87 \times 15,87]$

[5]

$25[0,9]$

Stainless Steel

10-32 Coaxial Jack

Side

10-32 Coaxial Plug

Epoxy

10-32 Female

10 to 20 [113 to 225]

NOTES:

[1] Zero based best straight line.

[2] Maximum without mass load.

[4] Resolution dependent on range setting and cable length used in charge system.

[3] Measured, mounted and unloaded.

[5] Hex $x$ height.

\begin{tabular}{|c|c|c|c|}
\hline Approved & $\mathrm{Hs}_{3}$ & $8 / 22 / 94$ & Spec No. \\
\hline Engineer & $\mathrm{C}$ & $218-1010-80$ \\
\hline Sales & $\mathrm{SGC}$ & $8 / 22 / 94$ & Sheet 1 of 1 \\
\hline
\end{tabular}




\section{SUPPLEMENTARY MATERIAL}

\section{Application of the stiffening frame}

4 The wooden supports were positioned while the treadmill was in an incline position (+10 grades). Marks were

5 drawn on the floor to define the lateral borders of the treadmill base. Wood supports were then placed as far

6 forward (front support) and backward (back support) as possible. The treadmill was then repositioned to flat

7 position, thus, the treadmill was resting on the wooded supports and not relying on its four legs. Pitch angle and

8 height from the floor was measured with and without supports using an electronic inclinometer and a calliper

9 respectively. Negligible differences in pitch $\left(+0.3^{\circ}\right.$ with, $-0.2^{\circ}$ without) and $17 \mathrm{~mm}$ difference (higher with support)

10 in height were measured. Difference in height was expected and intentionally done to have the treadmill fully

11 resting on the supports. Once the supports were in place we allow more than one hour before conducting the

12 experiment to account for any possible adjustment (wood compression), we then performed the hammer test again

13 to measure the effect of the wooden supports on the natural frequency of the system. Wood supports height was

14 measured along their length in different points, before and after the experiment which resulted in no differences

15 (450 mm before and after). 


\section{FFT Waveforms}

\section{FX \\ Resonant Frequency $=352$}
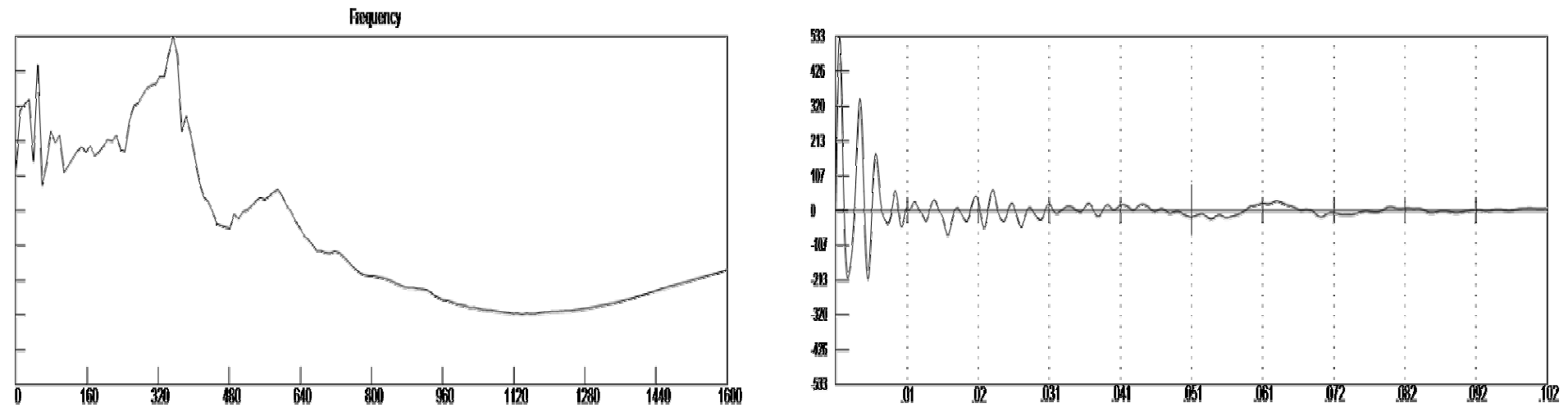

FY

Resonant Frequency $=352$
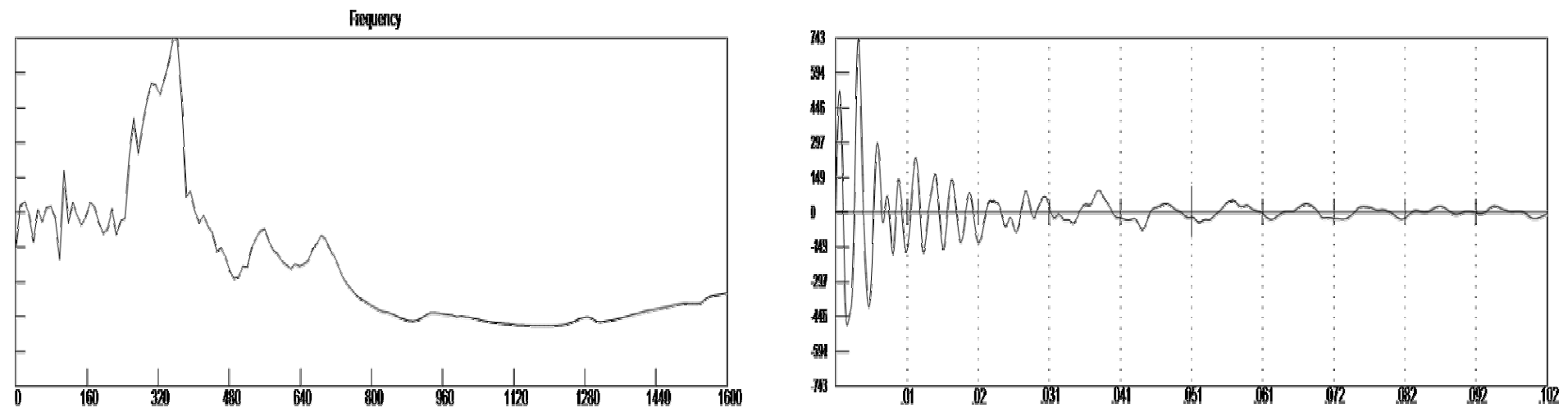

FZ

Resonant Frequency $=59$
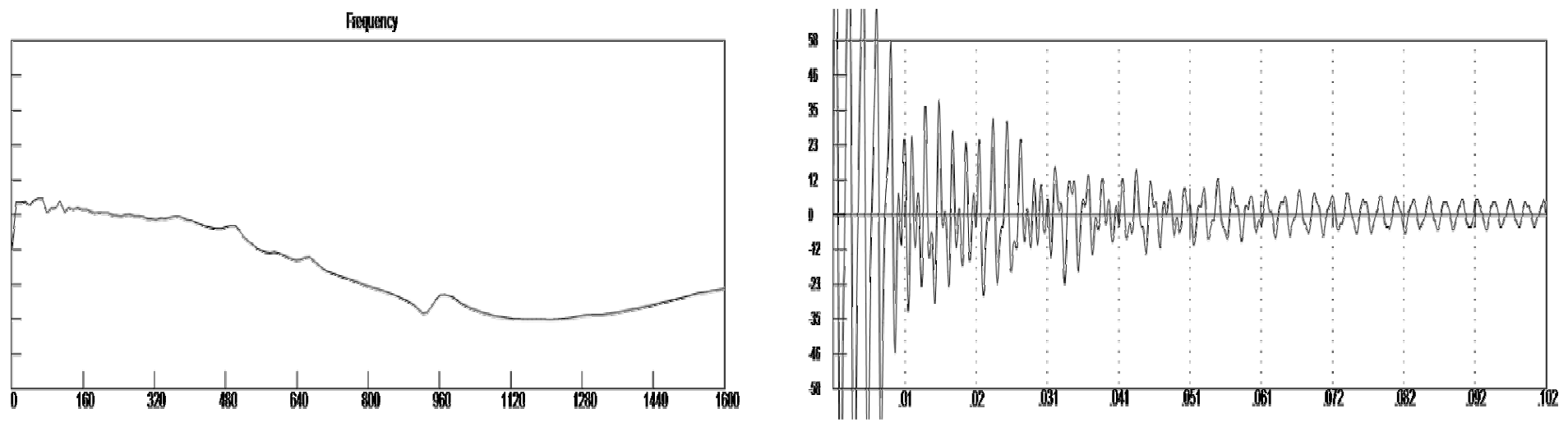